\title{
Impact of liver vasculature on electric field distribution during electroporation treatments: an anatomically realistic numerical study
}

\author{
R. Qasrawi ${ }^{1}$ and A. Ivorra ${ }^{1}$ \\ ${ }^{1}$ Universitat Pompeu Fabra/Department of Information and Communication Technologies, Barcelona, Spain
}

\begin{abstract}
Electroporation is the phenomenon in which cell membrane permeability is increased by exposing the cell to high intensity electric fields. In living tissues, such permeabilization boost can be used in order to enhance the penetration of drugs or DNA plasmids or to destroy undesirable cells and it is typically performed by applying pulsed high voltages across needle electrodes. When used for ablation, and particularly in the context of liver tumors, it is often claimed that in contrast with thermal ablation techniques, electroporation is not severely impacted by the presence of large blood vessels because the heat sinking characteristic of those is not relevant for the electric field distribution. However, blood vessels do distort the electric field distribution because of their high inner conductivity. The goal of the study presented here is to numerically analyze the relevance of such distortion in a clinical scenario. An anatomically realistic model of the liver and its vasculature within an abdominal section was implemented. The blood vessels ranged from 0.3 to $6.3 \mathrm{~mm}$ in diameter and from 24 to $68.56 \mathrm{~mm}$ in length. A series of simulations with plausible random locations and depths (needle tips from 10 to $25 \mathrm{~mm}$ within the liver) for the electrode pairs was conducted. Locations and depths were carefully verified so that the electrodes did not penetrate the vessels. The results were compared to those assuming that liver tissue is homogeneous. These comparisons indicate that volume error caused by neglecting the presence of vessels is less than $3 \%$, which can be considered negligible if compared to other error sources. However, it was noticed that if the treated region comprises vessels or is very close to them, undertreated and over treated spots will appear around the vessels. Since undertreated spots may imply that tumor cells remain viable after treatment, caution is advised.
\end{abstract}

Keywords- electroporation, conductivity, numerical modeling, blood vessels.

\section{INTRODUCTION}

Electroporation is the phenomenon in which cell membrane permeability to ions and molecules is increased after exposing the cell to high electric fields [1]. It requires placing electrodes within the target region and delivering a series of short pulses $(\sim 100 \mu \mathrm{s})$ of high electric field magnitude $(>100 \mathrm{~V} / \mathrm{cm})[2,3]$. The pulsed fields very significantly increase the cell transmembrane resting potential [1], and can result in two types of electroporation: reversible electroporation (RE), in which the permeabilized cell membrane completely reseals after minutes yielding viable cells, and irreversible electroporation (IRE), in which the cell membrane permeability is so severely increased that it disrupts cell homeostasis up to the point of causing cell death [1].

Tissue electroporation strongly depends on the distribution of the electric field magnitude. For a specific pulse protocol (i.e. number of pulses, shape, duration and frequency) it is generally considered that electroporation occurs at those locations where the electric field magnitude surpasses a specific threshold. Such electric field distribution depends on geometrical features (both of the electrodes and of the tissues), on tissue dielectric properties and on the electroporation phenomenon [4].

The electric field distribution can be predicted using predefined geometries (for the electrodes and tissues) or by performing numerical computation employing anatomical models [5] and knowledge about dielectric tissue properties and how those are impacted by electroporation [5-7].

When used for ablation, and particularly in the context of hepatic tumors, it is often claimed that in contrast with thermal ablation techniques, such as radiofrequency ablation, electroporation is not severely impacted by the presence of large blood vessels because the heat sinking characteristic of those vessels is not relevant for the electric field distribution [8]. In consequence, liver tissue is treated as a homogenous resistive material [9]. Blood vessels, however, do distort the electric field distribution because of their high inner conductivity. And the liver is considered as highly vascular organ; it contains large blood vessels, including hepatic veins, hepatic arteries, portal veins and hepatic ducts. The aim of this study is to provide an illustrative analysis about the effect of blood vessels on the electric field distribution in a realistic model that represents the treatment of liver tissues by means of electroporation. Up to our knowledge there are very limited studies on the impact of blood vessels on electroporation treatments [8].

\section{MATERIALS AND METHODS}

A realistic anatomical geometry of a male human liver, its blood vessels and the bones and surrounding tissues was used in our model. The 3D anatomical geometry was devel- 
oped by the Korea Institute of Science and Technology Information (KISTI) [11]. The geometry was decimated and prepared for simulation using the Graphical Interface for Medical Image Analysis and Simulation (GIMIAS) software developed by the Center for Computational Imaging and Simulation Technology in Biomedicine (CISTIB) at the Universitat Pompeu Fabra [12]. The main liver and vessels geometrical features are illustrated in table 1 . The geometry is shown in figure 1 together with one of the assayed probes locations. Each needle probe, with a diameter of $1 \mathrm{~mm}$, consisted of a passive shaft (length $=40 \mathrm{~mm}$ ) and an active part (the electrode) with a length of $10 \mathrm{~mm}$.

The 3D geometry was imported into COMSOL Multiphysics 4.3a (Stockholm, Sweden) and the electric field distribution was computed as described in [6] with a dynamic conductivity model for the liver which mimics the impact of electroporation on liver tissue conductivity. The entire geometry was meshed using the multiphysics regular refinement method user controlled tetrahedral mesh with minimum element size of $0.34 \mathrm{~mm}$. The total number of elements was 1567794 elements.

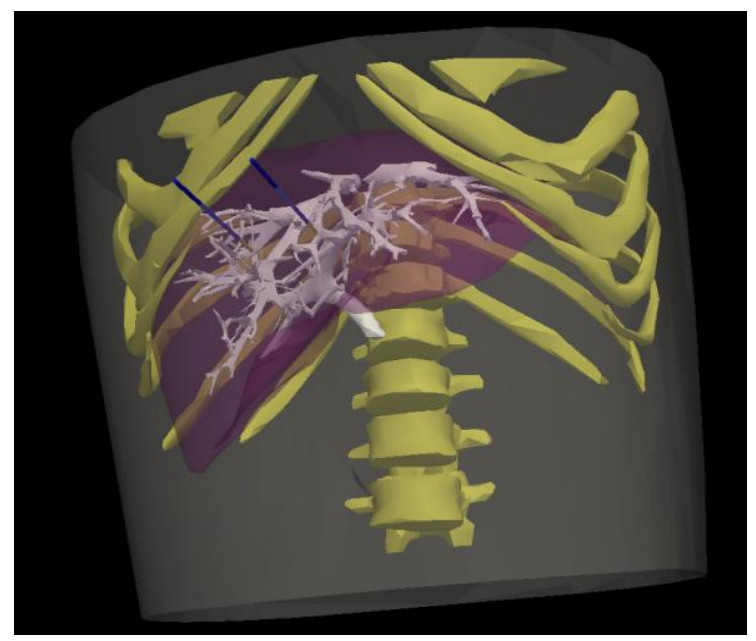

Fig. 1 The realistic anatomical 3D geometry representation of liver, vessels, bones, surrounding tissues and electrodes used in our model.

The initial conductivities for the liver, the vessels, the bones and surrounding soft tissues (a combination of muscle, fat, body fluids and epithelial layers) were $0.05 \mathrm{~S} / \mathrm{m}$, $0.7 \mathrm{~S} / \mathrm{m}, 0.02 \mathrm{~S} / \mathrm{m}$ and $0.2 \mathrm{~S} / \mathrm{m}$ respectively. These values were based on measurements reported elsewhere [5, 10]. The electric conductivity of the electrodes and insulators (passive shafts) were set to $1 \times 10^{5} \mathrm{~S} / \mathrm{m}$ and $1 \times 10^{-5} \mathrm{~S} / \mathrm{m}$ respectively. One of the electrodes is set to $0 \mathrm{~V}$ and the other to $\mathrm{V}_{0}=2000 \mathrm{~V}$. The boundaries of domains that are not in contact with electrode are treated as insulators.
The dependence of conductivity on the electric field magnitude (dynamic conductivity model) was modeled by the sigmoid function:

$$
\sigma_{\text {liver }}(|\mathbf{E}|)=\sigma_{0}+\left(\sigma_{f}-\sigma_{0}\right) \frac{1}{1+e^{-\frac{|\mathbf{E}|-E_{\text {th }}}{W}}}
$$

Where $\sigma_{0}=0.05 \mathrm{~S} / \mathrm{m}, \sigma_{\mathrm{f}}=0.3 \mathrm{~S} / \mathrm{m}, \mathrm{E}_{\mathrm{th}}=950 \mathrm{~V} / \mathrm{cm}$ and $W=200 \mathrm{~V} / \mathrm{cm}$.

Two electric field thresholds for electroporation were tried: $500 \mathrm{~V} / \mathrm{cm}$ and $1000 \mathrm{~V} / \mathrm{cm}$. These two values were deemed to be representative of the electroporation thresholds found in clinical electroporation protocols and, in particular, in irreversible electroporation for liver ablation.

A series of pseudorandom electrode positions and insertion depths were selected which would correspond to probe insertions through the anterior part of the abdomen. The insertion depths were $10,15,20$, and $25 \mathrm{~mm}$ (electrode tip depth within the liver). The distances between the two electrodes were 10 and $20 \mathrm{~mm}$. In all cases the two needles were parallel. The total number of cases was 48 (6 locations $\times 4$ depths $\times 2$ separation distances). Locations and depths for the electrodes were carefully verified so that the electrodes did not penetrate the vessels nor were in contact with them (distance $>0.5 \mathrm{~mm}$ ). In addition it was checked that the probes did not go through bone tissue.

Table 1: Liver and vessels geometrical measurements

\begin{tabular}{lcccc}
\hline Vessels & $\begin{array}{c}\text { Min } \\
\text { Diameter } \\
(\mathrm{mm})\end{array}$ & $\begin{array}{c}\text { Max } \\
\text { Diameter } \\
(\mathrm{mm})\end{array}$ & $\begin{array}{c}\text { Length } \\
(\mathrm{mm})\end{array}$ & $\begin{array}{c}\text { Volume } \\
\left(\mathrm{mm}^{3}\right)\end{array}$ \\
\hline Left vein & 0.64 & 5.11 & 46.43 & 378.3 \\
Left duct & 0.83 & 1.75 & 51.82 & 47.62 \\
Left artery & 0.4 & 1.2 & 26.67 & 17.94 \\
Middle vein & 1 & 6.3 & 43.12 & 376 \\
Right vein & 0.6 & 5.16 & 57.8 & 562.4 \\
Right duct & 0.3 & 1.45 & 24.0 & 28.8 \\
Right artery & 0.4 & 1.8 & 26.67 & 31.99 \\
Portal vein & 0.72 & 5.9 & 68.56 & 933.3 \\
Liver & - & - & 176.24 & 140600 \\
\hline
\end{tabular}

\section{RESUltS}

A comparison analysis is performed between the simulation results assuming the presence of vessels (non homogeneous) and the results obtained assuming that liver tissue is completely homogeneous $\left(\sigma_{\text {vessels }}=\sigma_{\text {liver }}\right)$.

\section{A. Undertreated spots:}

Figure 2 shows the electric field distribution for sample cases corresponding to $20 \mathrm{~mm}$ electrode depth. It can be observed that around a vessel (with a $3.1 \mathrm{~mm}$ diameter) 
there is a small region (spot indicated with a white arrow) in which the electric field magnitude is significantly lower than that obtained for the homogeneous case. Similar results were found in other cases in which vessels are within the region defined by the two electrodes or close to them. In some cases it was observed that the electric field magnitude was significantly higher at other spots around the vessels (but in all cases there were spots of low electric field in these same vessels.)
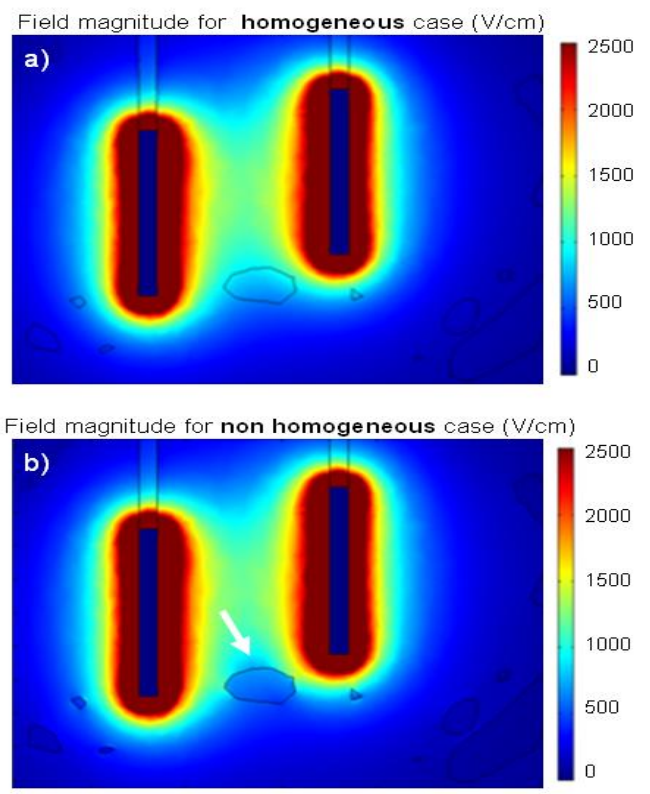

Fig. 2 Sample of electric field magnitude in the plane defined by the needles. The top subfigure (a) corresponds to a simulation in which liver tissue is assumed homogeneous while the bottom subfigure (b) corresponds to an equivalent simulation in which it is assumed the presence of vessels. The white arrow indicates a spot in which the electric field magnitude is significantly low.

Figure 3 shows the treated region $(|\mathrm{E}| \geq 1000 \mathrm{~V} / \mathrm{cm})$ for a sample case in which the electrodes are inserted $25 \mathrm{~mm}$ within the liver. It can be observed that the treated region is significantly distorted around the vessel.

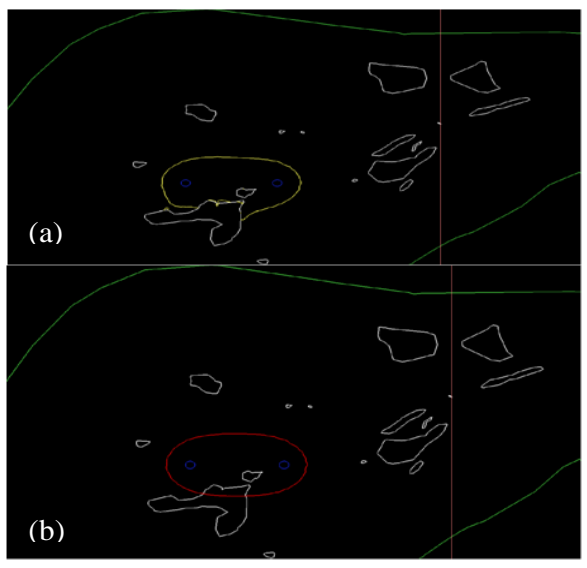

Fig. 3 Sample comparison between treated regions for the non homogenous case (a) and the homogenous case (b). The yellow line in (a) and the red line (b) indicate $|\mathrm{E}|=1000 \mathrm{~V} / \mathrm{cm}$. The treated volume (area in this cross section) is not significantly different between the two cases $(<1 \%)$ but, on the other hand, it can be noticed that the shape of the treated region is significantly distorted around the vessel.

\section{B. Impact of vessels on the treated volume}

The impact of vessels on the treated volume was assessed by estimating the average treated volumes of 6 random electrode positions with various insertion depths $(10,15,20$, and $25 \mathrm{~mm}$ ) for each position. In total 48 simulation cases were performed. Table 2 shows the average treated volumes of target tissues with different simulation parameters. The results indicate that there are no significant differences in the average treated volume. The relative difference in treated volumes between the two models was less than $3 \%$.

Table 2: Average treated volume of target tissues at 500 and $1000 \mathrm{~V} / \mathrm{cm}$

\begin{tabular}{cccccccc}
\hline & \multicolumn{4}{c}{$\mathbf{E} \geq \mathbf{5 0 0}$ V/cm } & \multicolumn{3}{c}{$\mathbf{E} \geq \mathbf{1 0 0 0}(\mathbf{V} / \mathbf{c m})$} \\
\hline $\begin{array}{c}\text { Spacing } \\
(\mathrm{mm})\end{array}$ & $\begin{array}{c}\text { Depth } \\
(\mathrm{mm})\end{array}$ & $\begin{array}{c}\mathrm{NH} \\
\left(\mathrm{cm}^{3}\right)\end{array}$ & $\begin{array}{c}\mathrm{H} \\
\left(\mathrm{cm}^{3}\right)\end{array}$ & $\begin{array}{l}\text { Max. } \\
\mathrm{D}(\%)\end{array}$ & $\begin{array}{l}\mathrm{NH} \\
\left(\mathrm{cm}^{3}\right)\end{array}$ & $\begin{array}{c}\mathrm{H} \\
\left(\mathrm{cm}^{3}\right)\end{array}$ & $\begin{array}{l}\text { Max. } \\
\mathrm{D}(\%)\end{array}$ \\
& 10 & 326.1 & 322.8 & 2.0 & 177.8 & 175.0 & 2.6 \\
& 15 & 304.5 & 299.5 & 2.1 & 165.9 & 162.7 & 2.8 \\
& 20 & 299.6 & 295.4 & 2.6 & 164.2 & 161.5 & 2.3 \\
& 25 & 303.4 & 297.9 & 2.9 & 168.7 & 165.9 & 2.6 \\
20 & 10 & 604.6 & 596.4 & 2.0 & 318.1 & 312.9 & 2.6 \\
& 15 & 576.3 & 573.1 & 0.9 & 293.7 & 291.0 & 1.7 \\
& 20 & 577.2 & 568.8 & 2.7 & 296.3 & 292.9 & 1.8 \\
& 25 & 541.2 & 533.4 & 2.5 & 309.0 & 303.8 & 2.3 \\
\hline
\end{tabular}

NH: Non Homogeneous. H: Homogeneous. Max. D is the maximum relative deviation between N.H. and $\mathrm{H}$ 


\section{DisCuSSION AND CONCLUSIONS}

By performing the comparison between the homogeneous and the non homogeneous cases, it can be concluded that liver blood vessels may have a significant impact on electroporation-based treatments. The undertreated (potentially sub-lethal) spots around medium size vessels cannot be considered as negligible in size (volumes above $1 \mathrm{~mm}^{3}$ ). Since undertreated spots may imply that tumor cells remain viable after treatment, caution is advised. However, it cannot be concluded that those untreated spots will necessarily yield new tumor nodules because in fact most likely they will be surrounded by ischemic tissue.

On the other hand, it must be pointed out that no significant differences were found in terms of treated volume size.

This study will be followed by a more exhaustive study in which deeper locations will be assayed and overall shape distortion will be quantified. In addition, more realistic models for the vessels (including endothelial and muscle layers) may be considered. Nevertheless, we do not expect that such modeling improvements will modify the main conclusion from this study. That is, the presence of blood vessels in liver does not alter significantly the treatment volume but it may cause sub-lethal regions.

\section{AcKNOWLEDGMENTS}

The authors would like to thank the Korea Institute of Science and Technology Information (KISTI) for their assistance in providing us with the anatomical geometry.

This work has received financial support from the Spanish Government through grant TEC2010-17285 "MEPVIVO" and from the European Commission through the Marie Curie IRG grant “TAMIVIVE" (256376).

\section{CONFLICTS OF INTEREST}

R. Q. and A.I. have no conflicts of interest to declare.

\section{REFERENCES}

[1] Weaver J (2003) Electroporation of biological membranes from multicellular to nano scales. IEEE T Dielect El In 10 754-768.

[2] Garcia P, Rossmeisl J, Davalos R (2011) Electrical conductivity changes during irreversible electroporation treatment of brain cancer. Conf Proc IEEE Eng Med Biol Soc 739-742.

[3] Edd J, Horowitz L, Davalos R, Mir L, and Rubinsky B (2006) In vivo results of a new focal tissue ablationtechnique: irreversible electroporation. IEEE Trans Biomed Eng 53 1409-15.

[4] Ivorra A, Al-Sakere B, Rubinsky B, Mir L (2009) In vivo electrical conductivity measurements during and after tumor electroporation: Conductivity changes reflect the treatment outcome. Phys. Med. Biol. vol.54, pp.5949-5963.

[5] Miklavcic D, Snoj M, Zupanic A, Kos B, Cemazar M, Kropivnik M, Bracko M, Pecnik T, Gadzijev E, Sersa G (2010) Towards treatment planning and treatment of deep-seated solid tumors by electrochemotherapy. BioMedical Engineering OnLine, 9, 10.

[6] Ivorra A, Villemejane J, Mir L (2010) Electrical modeling of the influence of medium conductivity on electroporation. Phys. Chem. Chem. Phys., vol. 12, pp.10055 -10064.

[7] Miklavcic D, Pavselj N, Hart F (2006) Electric Properties of Tissues. In Wiley Encyclopedia of Biomedical Engineering John Wiley \& Sons, New York.

[8] Lee Y, Lu D, Osuagwu F, Lassman C (2012) Irreversible electroporation in porcine liver: short- and long-term effect on the hepatic veins and adjacent tissue by $\mathrm{CT}$ with pathological correlation. Invest Radiol 47(11):671-675

[9] Narayanan G. (2011) Irreversible electroporation for treatment of liver cancer. Gastroenterol Hepatol 7(5):313-316.

[10] Andreuccetti D, Fossi R, Petrucci C (1996) An Internet resource for the calculation of the dielectric properties of body tissues in the frequency range $10 \mathrm{~Hz}-100 \mathrm{GHz}$. Website at http://niremf.ifac.cnr.it/tissprop/. IFAC-CNR, Florence, Italy.

[11] Shin D, Chung M, Lee J, Park J, Chung J, Lee S, Park J, Chung J, Lee S, Lee S (2009) Advanced surface reconstruction technique to build detailed surface models of liver and neighboring structures from the Visible Korean Human. J Korean Med Sci 24: 375-383.

[12]Larrabide I, Omedas P, Martelli Y, Planes X, Nieber M, Moya J, Butakoff C, Sebastián R, Camara O, Craene M, Bijnens B, Frangi F(2010) Gimias: An open source framework for efficient development of research tools and clinical prototypes. Func Imaging and Modeling of the Heart, ser. Lecture Notes in Computer Science, 5528:417-426.

$\begin{array}{ll}\text { Author: } & \text { Radwan Qasrawi } \\ \text { Institute: } & \text { Universitat Pompeu Fabra } \\ \text { Street: } & \text { Roc Boronat } 138 \\ \text { City: } & \text { Barcelona E-081018 } \\ \text { Country: } & \text { Spain } \\ \text { Email: } & \text { qasrawir@gmail.com }\end{array}$

